\title{
LE RATEAU FAUCARDEUR RELEVABLE
}

\author{
par J. ARRIGNON (1) et J. JUSTINE (2)
}

avec la collaboration technique d'A. RUMEAU (3)

\section{RESUME}

Un rateau monté sur deux bras mobiles à l'avant d'un bateau faucardeur permet de récupérer les végétaux coupés et de les déposer sur la berge.

Le positionnement de la grille horizontale permet en outre l'enlèvement en profondeur des végétaux mous et notamment des algues filamenteuses ainsi que la récupération en surface des lentilles d'eau.

La simplicité de la conception, la légèreté de l'ouțil, sa maniabilité et son coût modiạue s'associent à son efficacité pour le désigner comme une solution séduisante du problème de l'enlèvement des lentilles d'eau et des produits de faucardement dans les étangs et canaux.

\section{I. - INTRODUCTION}

Les gestionnaires de plans d'eau et de canaux sont souvent confrontés avec deux problèmes d'entretien: la récupération des végétaux faucardés et la lutte contre la prolifération des lentilles d'eau.

1.1. - La récupération des végétaux coupés lors des faucardements, est très irrégulièrement effectuée, bien qu'elle soit réglementairement obligatoire dans les eaux libres.

(1) Ingénieur Chargé de la Région Piscicole de COMPIEGNE au CONSEIL SUPERIEUR de la PECHE : 3, rue Sainte-Maric - 60200 COMPIEGNE.

(2) Président de la Société de Pêche de MAISON BLANCHE : Avenue Jules-Verne, 93220 GAGNY.

(3) Garde-Pêche Commissionné à la Région Piscicole de COMPIEGNE.

Article available at http://www.kmae-journal.org or http://dx.doi.org/10.1051/kmae:1976009 
Cette négligence, due aux difficultés de l'opération conduit à des phénomènes de transport et d'accumulation désagréables pour les riverains et pour les usagers de l'eau, dont'les pêcheurs.

En étangs et dans les canaux, les conséquences passent souvent du simple désagrément à l'accident fatal. En raison de l'absence de courant d'eau dans certains canaux et en raison du milieu réduit et fermé des étangs, les produits de la décomposition des végétaux faucardés, loin d'être dispersés, sont concentrés. II s'en suit une pollution organique par suite de la mobilisation de l'oxygène indispensable à la faune aquatique et de la libération de produits de décomposition qui sont nuisibles à cette faune : les mortalités de poissons subséquentes sont fréquentes et dommageables en période estivale.

1.2. - La lutte contre la prolifération des lentilles d'eau (Lemna sp.) est tout aussi difficile et son imperfection est également génératrice de déboires.

Les plages de lentilles, discontinues, errant au gré du vent, ou le tapis continu qui recouvre étangs et canaux constituent un désagrément immédiat et certain pour le pêcheur à lá ligne dans l'exércice de son sport.

Les inconvénients occultes sont encore plus graves que les désagréments visibles : le tapis de lentilles d'eau empêche la pénétration des rayons solaires dans la masse.liquide; il empêche également tout contact superficiel entre l'air et l'eau. Le cycle biologique très court en été fait que meurent des masses de lentilles sans cesse renouvelées qui, en raison de ce qui précède, trouvent fort peu d'oxygène dans l'eau pour être dégradées et constituent ainsi une pollution potentielle d'autant plus grave qu'elle se manifeste brutalement non pas toujours en été, mais souvent en fin d'automne et encore bien davantage au tout début du printemps suivant.

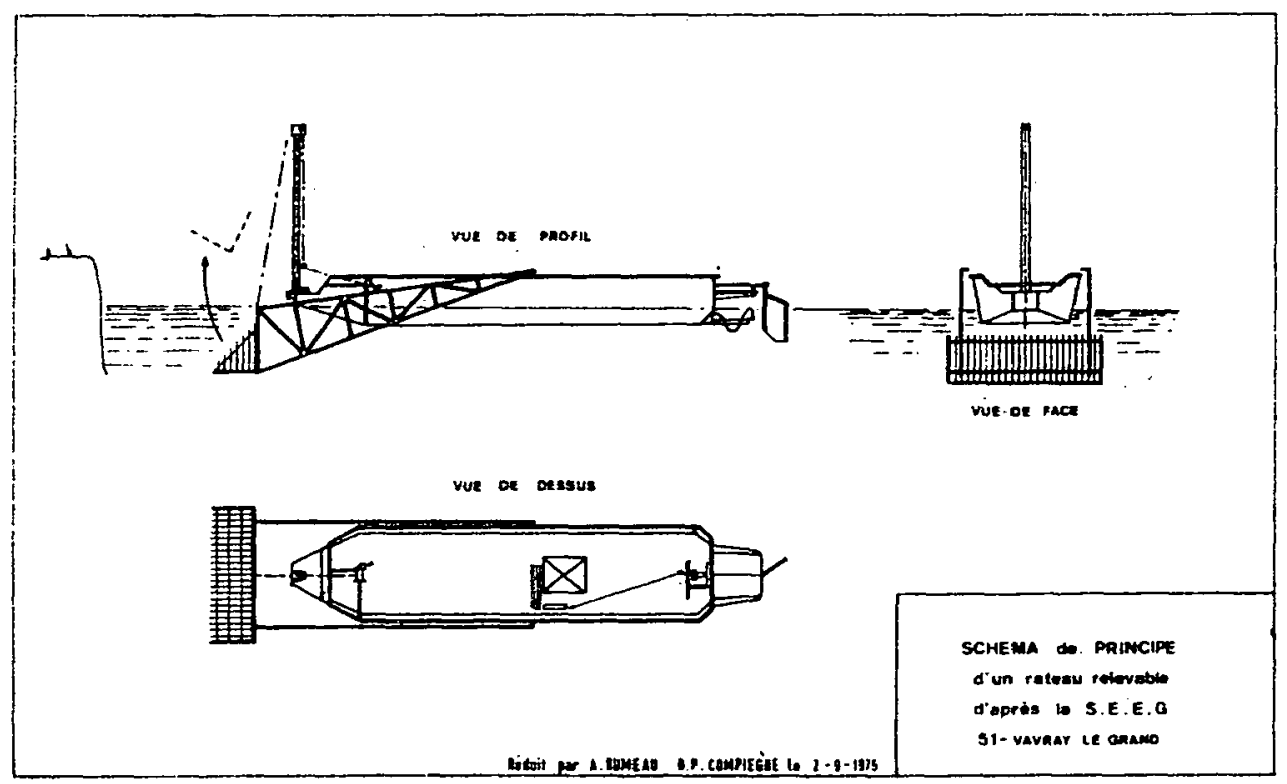




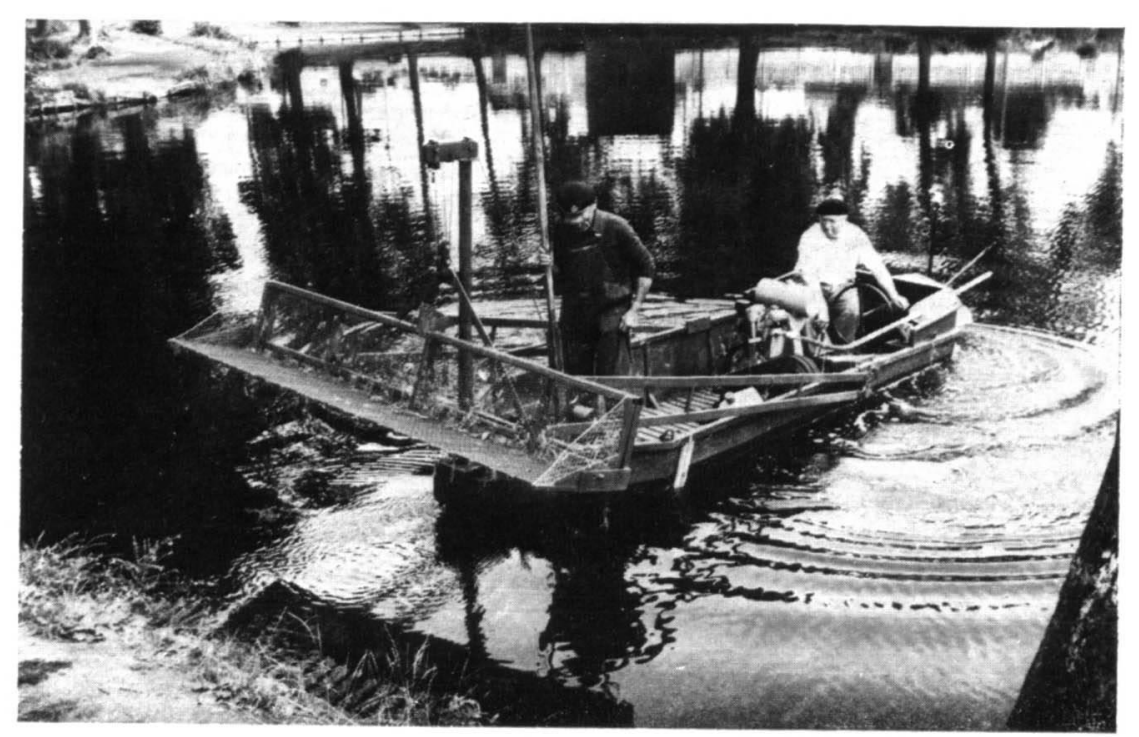

Clichè no 1. - J. ARRIGNON.

Présentation du rateau faucardeur relevable, en position haute, monté sur un bateau faucardeur GIBEAUX.

Etang de MAISON-BLANCHE. Société de Pêche de GAGNY (Seine-Saint-Denis).

\section{3. - Les solutions}

Le premier problème a trouvé jusqu'alors sa solution dans des travaux de récupération manuelle, longs et épuisants.

La solution du second, en dehors de l'action directe des oies et canards consommateurs de Lemna, a davantage été recherchée dans l'intervention de la chimie que dans des amendements, et autres interventions tendant à modifier les caractéristiques du milieu, sans toutefois que des succès appréciables aient pu être enregistrés avec les divers désherbants chimiques employés (Dichlobenil - 2, 4 , D, etc.).

Difficultés et échecs ont orienté la recherche de solution vers une solution mécanique, faisant appel à un matériel assurant l'enlèvement des végétaux coupés aussi bien que celui des lentilles d'eau.

Ce matériel a été trouvé dans la gamme des PELICANS, conçus pour nettoyer les ports et les plages des hydrocarbures et corps. flottants divers (Service de l'Equipement - Département de CHARENTE-MARITIME). II a également été trouvé dans les accessoires des bateaux faucardeurs : c'est ainsi que leś Etablissements GIBEAUX ont conçu un rateau faucardeur relevable, amélioré par Jean JUSTINE. Ce matériel, actuellement fonctionne! et opérationnel, est décrit ci-après. 


\section{2. - DESCRIPTION}

\section{1. - Le panier}

Le rateau faucardeur relevable s'adapte frontalement sur un bateau faucardeur de type GIBEAUX (voir croquis et clichés.)

De même que la pelle d'un bulldozer, il est monté sur deux bras articulés latéralement sur chaque bordé. En raison du jeu latéral existant entre bordé et bras, il est nécessaire de prévoir un calage de rattrapage entre la coque è le bordé de façon à ce que le bras n'accroche pas ce dernier lors des manipulations (cliché $n^{\circ} 1$. pièce blanche en regard de l'étrésillon du bras gauche).

Solidaire des deux bras, une armature métallique reçoit le panier proprement dit qui, monté de chaque côté sur des pivots, peut basculer sous l'action d'un levier (cliché $n^{\circ} 5$ : l'opérateur. a encore la main sur le levier; l'inclinaison du rateau est réglée par une chaine).

Le panier est constitué par un cadre frontal et un cadre horizontal soudés à angle droit. Le cadre inférieur est renforcé de façon à pouvoir supporter sans déformation la charge de végétaux récupérés.

A cet effet, les' deux cadres sont garnis d'un treillagé métallique dont les mailles sont choisies en fonction du calibre des végétaux à récupérer (herbes faucardées ou lentilles d'eau). Les joues du panier sont matérialisées par le même treillage (cliché $n^{\circ} 1$ ).

\section{2. - Le système de levage}

Le système de levage est assuré par une potence métallique solidaire de la proue de l'embarcation ainsi que par un jeu de moufles démultipliant un treuil à manivelle fixé à l'avant du bateau. Le nombre de moufles est fonction des caractéristiques du panier et par conséquent du poids maximal des végétaux susceptibles d'être enlevés lors de chaque passe.

\section{3. - L'embarcation}

Il est utile de souligner que l'embarcation doit être lourde, stable mais d'une grande maniabilité. Le porte à faux, lors du levage du panier à pleine charge, est important; il doit être compensé par une excellente assiette de l'embarcation et notamment par un centre de gravité très décalé à l'arrière.

Il convient de remarquer qu'un bateau faucardeur est conçu pour admettre à l'avant un porté à faux important constitué par le dispositif de faucardage et que le rateau relevable prend la plaee en quelque sorte de ce dispositif. Le bateau.faucardeur parait, en l'état actuel des choses, le plus approprié pour recevoir un rateau relevable avec les meilleures chances d'opérer convenablement.

L'équipement d'une émbarcation autre qu'un bateau faucardeur requièrera de la part du monteur une étude sérieuse des caractéristiques de l'embarcation, de son assiette et de la répartition des charges. 


\section{4. - Caractéristiques}

Les caractéristiques du rateau faucardeur relevable sont fonction des caractéristiques de l'embarcation porteuse. L'amplitude entre position basse et position haute du panier dépend de la stabilité et de l'assiette de l'embarcation.

Les clichés représentent un panier basculant grillagé de $2,50 \mathrm{~m}$ de largeur sur $0,50 \mathrm{~m}$ de profondeur et $0,40 \mathrm{~m}$ de hauteur frontale.

La longueur des bras est de $2,80 \mathrm{~m}$; l'amplitude est de $2,40 \mathrm{~m}$ ce qui signifie que le relevage au-dessus du niveau d'eau est de 1,20 m environ.

L'embarcation porteuse mesure $5 \mathrm{~m}$ de long et $1,35 \mathrm{~m}$ de large.

\section{3. - FONCTIONNEMENT}

L'outil, décrit, ci-avant, assure trois fonctions :

- L'enlèvement de végéțaux aquatiques (ou de débris).

- Le transport de ces végétaux.

- Leur dépôt sur berge.

Le transport des végétaux impose à l'embarcation une allure lente en raison de la nature des produits transportés. II est souvent nécessaire de lever le panier lors du transport afin d'éviter une dispersion des végétaux sous l'action du courant.

Le dépôt sur berge requiert le choix de sites d'un abord aisé et d'une hauteur correspondảnt à la hauteur de levage du panier. Le nombre dés dépôts peut correspondre au nombre de passes lorsque la configuration de l'étang ou lorsque la largeur du canal le permettent.

L'enlèvement des végétaux est accompli différemment suivant qu'il s'agit de l'arrachage d'herbes molles (Myriophyllum sp., Ceratophyllum sp., Potomageton ep. Elodea, etc.), de l'enlèvement d'algues filamenteuses (Cladophora, Spirogyra; etc.) ou de la récupération de lentilles d'eau.

\section{1. - Arrachage des herbes molles}

Chaque passe doit, de préférence, porter sur de petites surfaces, de façon à ce que le panier ne soit pas surchargé. En position basse, le rateau ne doit décaper au niveau du fond que dans la mesure où la mise en mouvement de la vase n'aura aucun effet toxique à l'égard des poissons. C'est ainsi qu'en été il sera préférable d'attaquer les herbiers à mi-hauteur. 


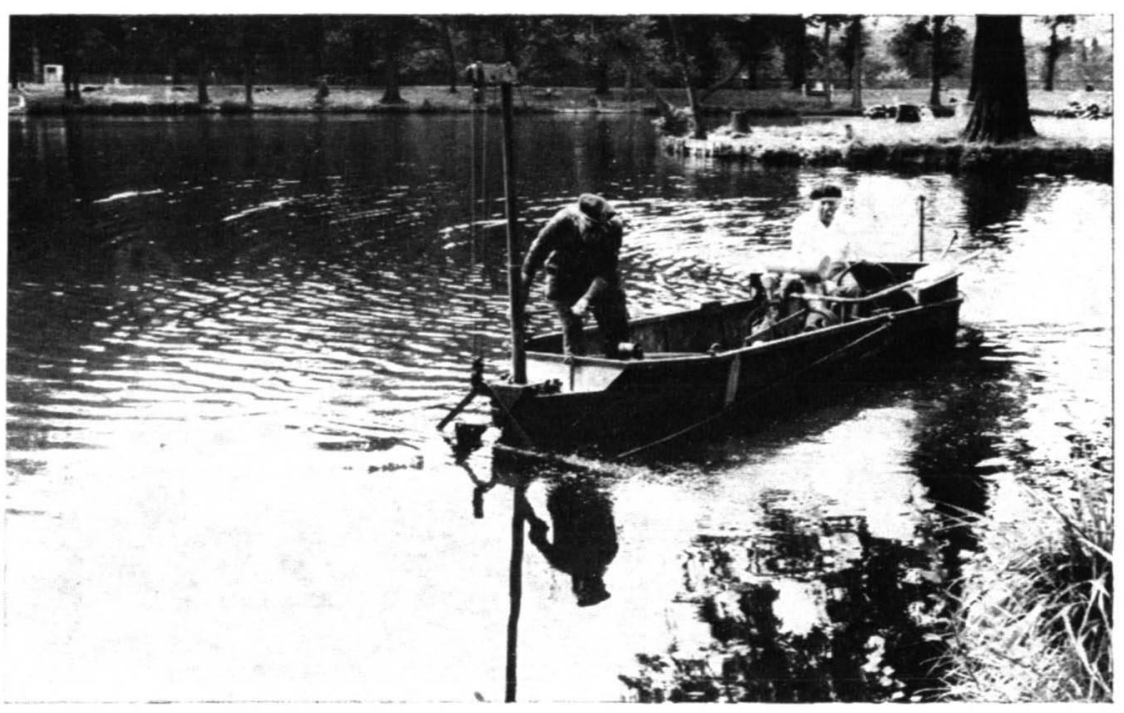

Cliché no 2. - ARRIGNON.

Rateau immergé, en cours de faucardage de végétaux aquatiques mous. Le panier est en position basse, l'opérateur surveille la récupération, le pilote veille à' ce qu'une frange végétale subsiste près de. la rive.

Etang de MAISON-BLANCHE. Société de Pêchè de GAGNY.

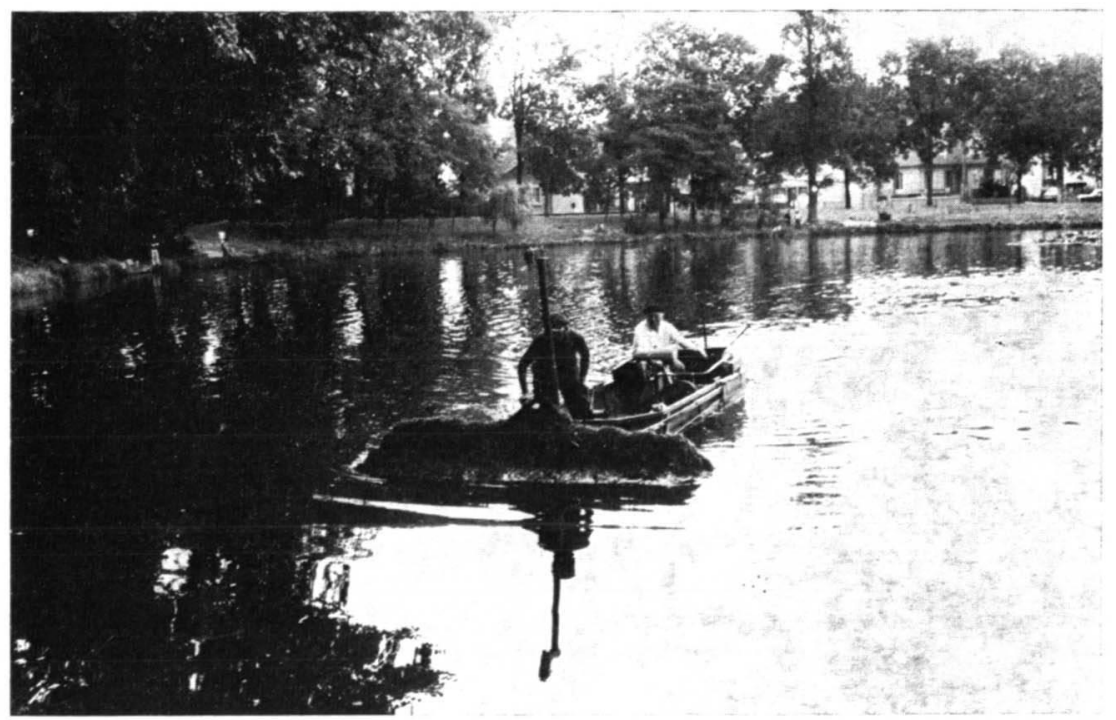

Cliché no 3. - J. ARRIGNON.

En fin de boucle, le bateau aborde la berge; le rateau est plein et relevé pour éviter la dispersion des végétaux recueillis.

Etang de MAISON-BLANCHE. Société de Pêche de GAGNY. 


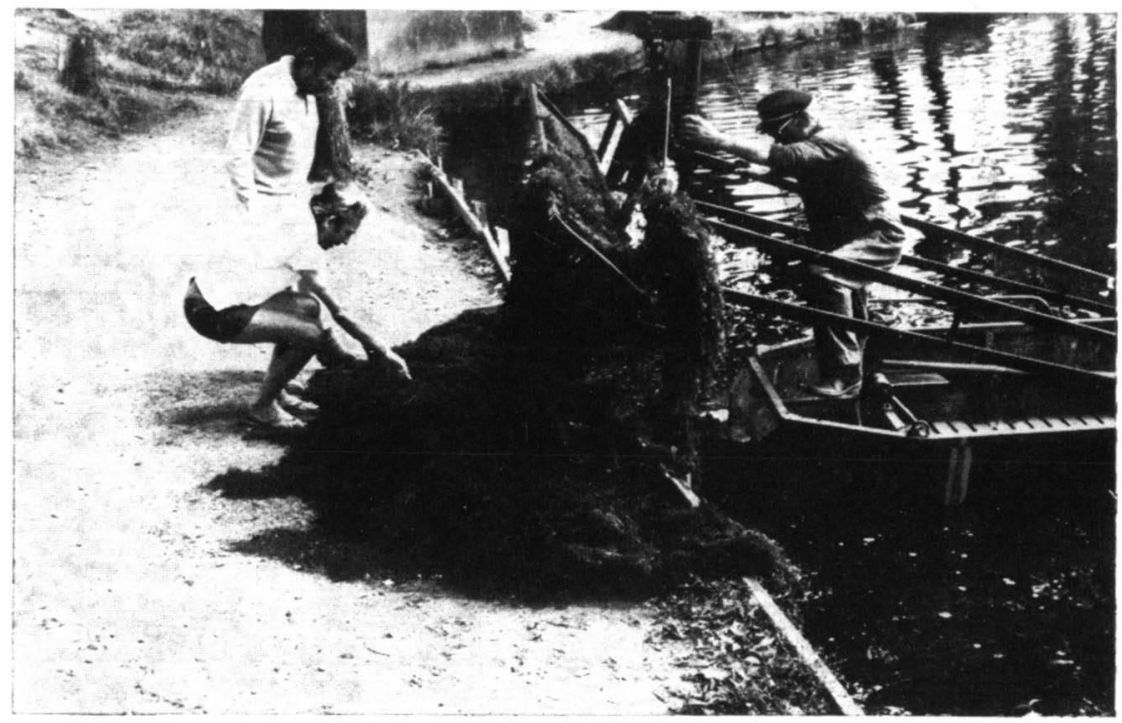

Cliché $n^{\circ} 4 .-$ J. ARRIGNON.

Rateau levé largement au-dessus de la berge, l'opérateur déclenche le basculement du panier en agissant sur le levier. Le jeu du panier est réglé par une chainette.

Etang de MAISON-BLANCHE. Société de Pêche de GAGNY. 


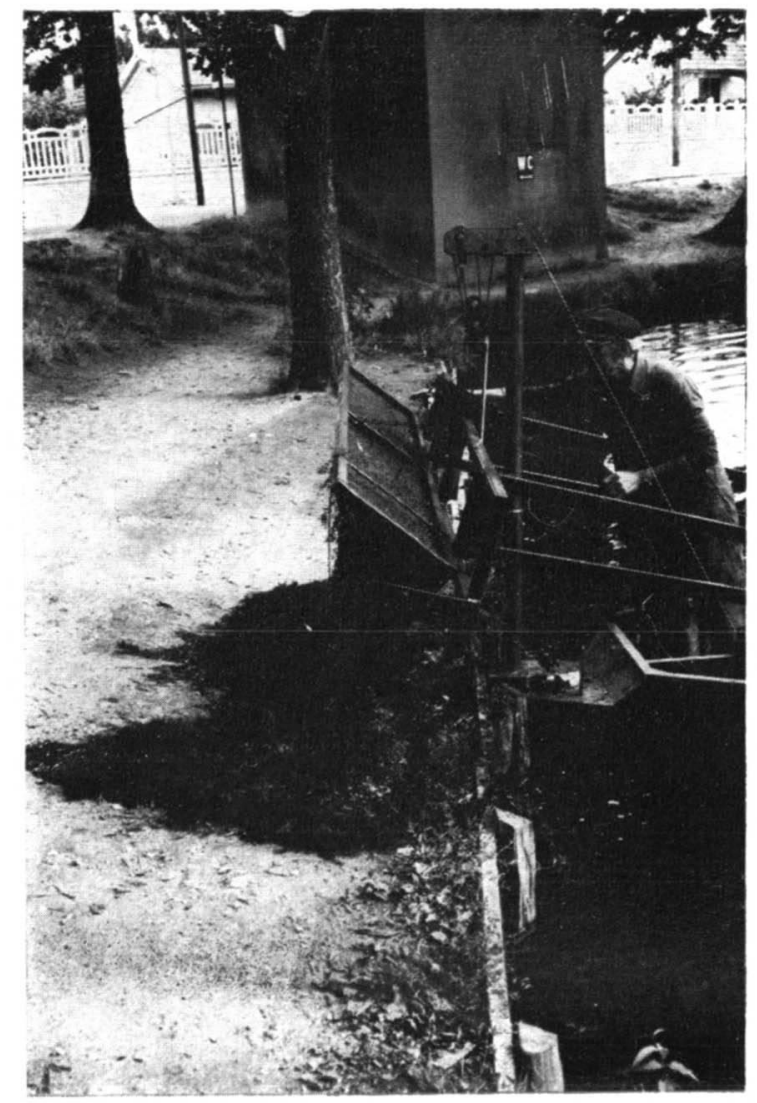

Cliché $n^{\circ}$ 5. - 1. ARRIGNON.

Les herbes sont en dépôt. On voit les détails du dispositif de levage et de basculement du panier.

Etang de MAISON-BLANCHE. Socièté de Pêche de GAGNY. 


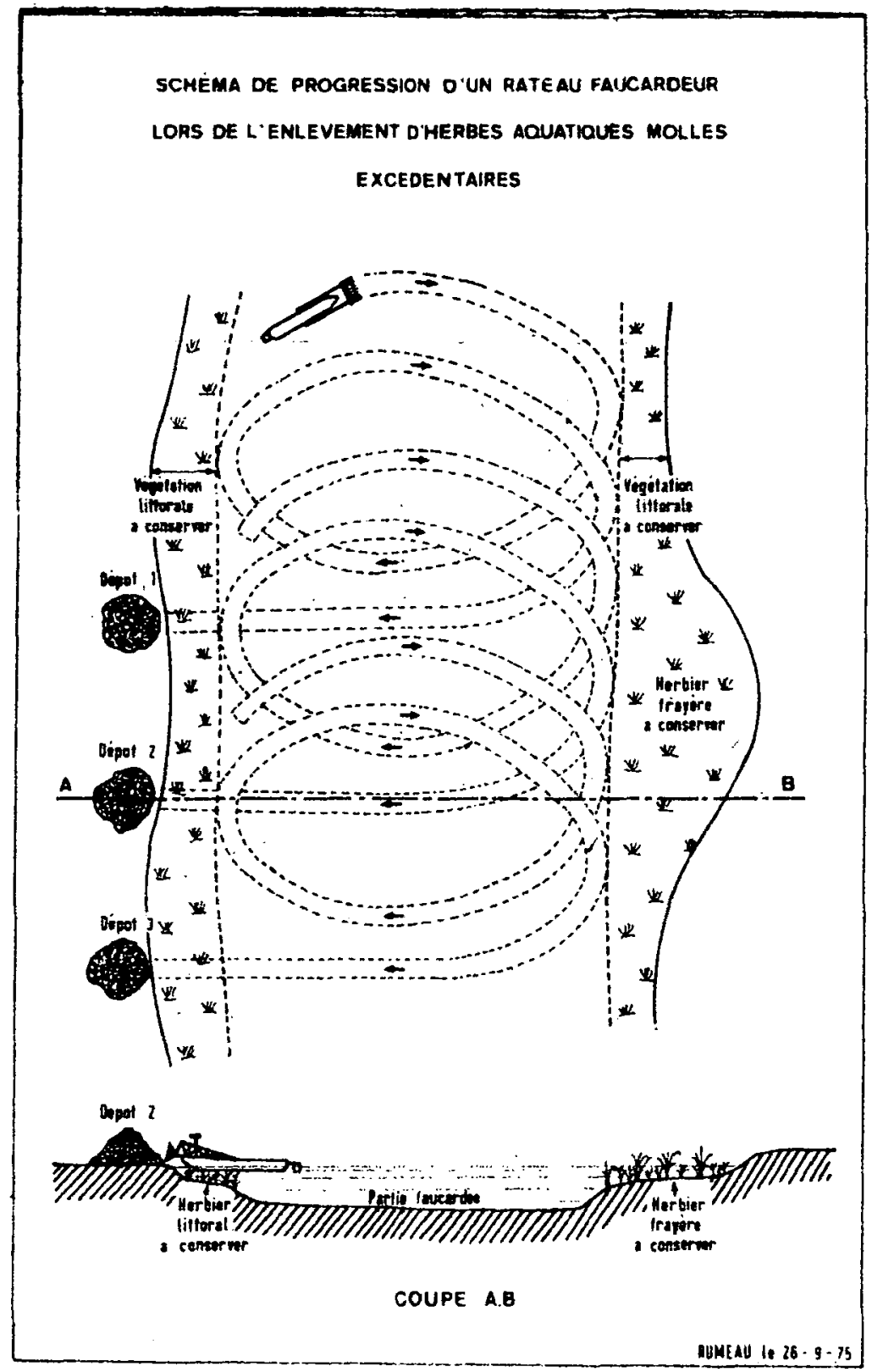


Suivant l'importance des herbiers aquatiques à dégager la passe sera droite (herbiers de faible importance) ou en spirale plus ou moins fermée (herbiers continus).

En se repérant sur les bords et en calant le gouvernail dans la même position (sauf pour avancer en direction du secteur à faucarder) on arrive aisément à opérer en spirale (Croquis $n^{\circ} 2$ ).

Pour obtenir un nettoyage correct, il est nécessaire de passer deux fois: une première pour faucarder les herbiers, une seconde pour récupérer ce qui a pu échapper au pänier lors du transport.

\section{2. - Enlèvement des algues filamenteuses}

L'opération doit être conduite très doucement de manièrè à laisser le temps à l'eau de traverser les algues récupérées par le rateaü. Une trop grande vitesse entraine la plus grande partie des algues hors du panier.

Le mode opératoire est celui décrit en (3.1.).à ceci près que la quantité d'algues évacuée à chaque passe est sensiblement moins importante que celle des végétaux supérieurs en raison précisément du colmatage des mailles du panier et des risques d'entrainement hors du panier sous la pression de l'eau.

\section{3. - Récupération des lentilles d'eau et des débris flottants}

Le rateau travaille à $10 \mathrm{~cm}$ environ sous la surface de l'eau de façon à " écrémer "les lentilles. En fin de pasśe la vitesse de l'embarcation doit être réduite en raison, là encore, des risques de dispersion des lentilles sous la pression frontale de l'eau. Il est préférable de faire des passes droites, les spires provoquant la dispersion des bancs de lentilles.

La récupération des débris flottants ne pose des problèmes que dans la mesure où ces débris, très légers, peuvent être chassés hors du panier par l'action du courant d'eau (bouteilles vides en matière plastique). L'enlèvement des roseaux et joncs faucardés à la lame est aisé.

\section{4. - CONCLUSION}

Deux personnes suffisent à assurer le fonctionnement, l'une de l'embarcation, l'autre du rateau. II est néanmoins souhaitable de prévoir un troisième opérateur pour le nettoyage du panier après dépôt sur berge (notamment lorsqu'il s'agit d'algues filamenteuses).

D'un coût modeste (2500 francs à la date de la rédaction du présent article) le rateau faucardeur relevable semble répondre aux besoins de nombreuses Sociétés de Pêche et de nombreux particuliers ayant des difficultés pour enlever les produits de faucardement et pour se débarrasser des lentilles d'eau.

La nature du matériel, la simplicité de son emploi sont à la mesure des moyens des gestionnaires locaux d'étangs et de canaux.

Les Etablissements GIBEAUX à VAVRAY-LE-GRAND, 51300 -VITRY-LEFRANÇOIS - Tél. (26) 41.31 .02 şont à même de donner tout complément d'information sur la fourniture de ce matériel. 